\section{El Método Científico: Análisis de la literatura}

\section{The Scientific Method: Analysis of the literature}

\section{Jhonnatan Chinche Duicela}

Estudiante, Escuela Superior Politécnica de

Chimborazo, Riobamba, Ecuador,

jhonnatan.chinche@espoch.edu.ec,

https://orcid.org/o0oo-0001-8130-5700

\section{Jenniffer Ramón Pozo}

Estudiante, Escuela Superior Politécnica de Chimborazo, Riobamba, Ecuador, jenniffer.ramon@espoch.edu.ec, https://orcid.org/oooo-0003-4001-1903

\section{José Fernando López Aguirre}

Magister en Administración y Dirección de

Empresas, Escuela Superior Politécnica de

Chimborazo, Riobamba, Ecuador,

jfo731@gmail.com, https://orcid.org/oooo-ooo19706-5115

\section{Resumen}

La presente investigación parte de la necesidad de conocer más a fondo sobre el método científico, empezando desde su evolución que ha tenido con los diferentes autores o investigadores como Isaac Newton quien con sus aportaciones en la ciencia han ayudado al desarrollo de la humanidad, dentro de esta búsqueda que se ha realizado sobre el método científico encontramos importante contenido como son las técnicas que se utilizan para el estudio del método, las cuales nos facilitaran la realización y comprensión de ciertos asuntos, otro de los temas incluidos en esta búsqueda de información son las

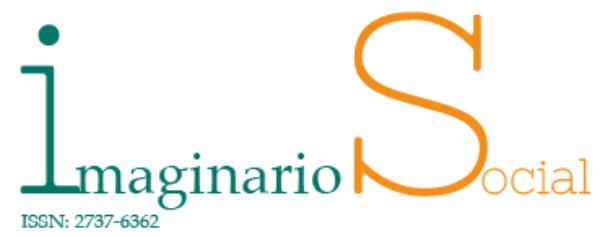

Imaginario Social e-ISSN: $2737-6362$

julio - diciembre 2020 Vol. 3-2-2020 http://revista-

imaginariosocial.com/index.php/es/in dex

Recepción: 2 de junio 2019

Aceptación: 19 de febrero 2020

$52-63$

Atribución/Reconocimiento-NoCo mercial- Compartirlgual 4.0 Licencia Pública Internacional - CC

BY-NC-SA 4.0

https://creativecommons.org/licenses /by-nc- sa/4.0/legalcode.es 
fases o etapas, las cuales mediante el desarrollo de cada una de estas nos da como resultado la resolución de un problema o interrogante que tiene la sociedad, luego de realizar toda la investigación se da a conocer en el desarrollo ciertos errores o problemas que surgen al usar esta metodología, para la obtención de información acerca de este tema investigativo se usó la metodología estratégica de revisión bibliográfica y una lectura crítica.

Palabras clave: Método científico, investigación, técnicas, información, metodología.

\begin{abstract}
The present investigation starts from the need to know more in depth about the scientific method, starting from its evolution with the different authors or researchers like Isaac Newton who with their contributions in science have helped the development of humanity, within this search that has been carried out on the scientific method we find important content such as the techniques that are used for the study of the method, which will facilitate the realization and understanding of certain issues, Another of the topics included in this search for information are the phases or stages, which through the development of each of these results in the resolution of a problem or question that society has. After carrying out all the research, certain errors or problems that arise when using this methodology are made known in the development.
\end{abstract}

Key word: Scientific method, research, techniques, information, methodology.

\title{
Introducción
}

Para poder entender de lo que trata el método científico es necesario empezar conociendo sus antecedentes, donde mediante la investigación que se ha realizado, se entiende que este método inicia con el hombre primitivo. (Junta de Andalucía, 2012) considera que la aparición del hombre en la tierra fue el primer paso para el nacimiento del pensamiento y un progreso hacia el desarrollo de la ciencia y la tecnología, debido a que años atrás para el ser humano todo era desconocido y sentía curiosidad por conocer el porqué de su existencia o uso, esto lo llevó a descubrir y 
desarrollar lo que le convenía o no, en cuanto a que comer y qué hacer, todo esto debido a repetidas experiencias que lo llevaron a seleccionar los frutos comestibles y a escoger sus refugios para sobrevivir

Surgieron entonces científicos como Aristóteles, creador de la Biología Zoología, Botánica, Anatomía y otras muchas ciencias, pasando al 1550 apareció Galileo Galilei quien destruyó los argumentos de Aristóteles mediante su inexorable método experimental, ratificando la conclusión con la experiencia, a esta lista se suma Roger Bacon quien está considerado como el precursor del método inductivo-experimental e Isaac Newton, con él la ciencia y el método científico ascendieron a alturas nunca obtenidas (Junta de Andalucía, 2012)

En siglo XIX luego de una carrera de descubrimientos hasta llegar el siglo XX aparece Albert Einstein quien añadió al método científico la ultra precisión y la ultra exactitud utilizando medidas tan precisas como la velocidad de la luz (González, A. M., \& Hernández Alegría, A., 2014), se puede observar que cada aporte e investigación al método científico realizada ha sido el producto de muchas mentes brillantes que han aportado lo mejor de sus conocimientos, observaciones y destrezas para el bien de la humanidad. Los aportes de cada uno de estos científicos nos han llevado a determinar que el método científico es un proceso de investigación que es usado como prioridad en la generación del conocimiento en la ciencia, ya que por medio de este método podemos tener una idea de lo que pasa en nuestro alrededor, y mediante esto tratar de solucionar ciertos problemas naturales existentes. (Castán, 2012), también podemos mencionar que cuenta con técnicas que son inductivo, deductivo, hipotéticodeductivo, analítico, sintético, histórico comparativo, cuantitativo y cualitativo cada una de estas técnicas se utiliza para el estudio del método científico (Asens \& Parra, 2002).

En cuanto a sus etapas o fases, cualquiera que sea la división del procedimiento, con la ayuda de las técnicas de investigación ya mencionadas, deben superar las siguientes: identificación y definición del problema; recogida y tratamiento de los datos para su interpretación y difusión de los resultados obtenidos. Este método administra todo movimiento científico desde el nacimiento de la interrogante hasta la demostración de los resultados que se obtiene de dicha investigación. Para finalizar podemos decir que 
este método atreves de sus instrumentos tiene como resultado indicar y experimentar, las diferentes problemáticas que existen o que han ocurrido desde el origen de la vida hasta la actualidad, dando como resultado a cada una de estas problemáticas las posibles soluciones a la necesidad de la humanidad; según Canaan (2020) este método ayuda a disminuir los errores y brinda tranquilidad a los científicos por la seguridad y exactitud de sus resultados.

\section{Materiales y Métodos}

Antes de empezar cabe destacar que el método científico, tanto empírica como científicamente es el primer paso hacia el desarrollo de soluciones de la vida cotidiana y el desarrollo de conocimiento en las ciencias. Para Bungue (1967) el método científico es indispensable para hacer ciencia, por su parte Labajo (2016) infiere en que es un método (...) que se basa en leyes deducidas por el hombre (...) se construye estableciendo relaciones entre observables y no a partir de certezas absolutas. (...) y utiliza las Matemáticas para establecer la relación entre las variables. Además, afirma que está basado en 2 pilares fundamentales: la reproductibilidad, es decir la capacidad de poder repetirse varias veces; y la refutabilidad, que presenta al postulado como sujeto de cambio; por otra parte (Pollit \& Hungler, 1991) consideran que el MC es un estudio sistemático, controlado, empírico, reflexivo y crítico de proposiciones hipotéticas sobre las supuestas relaciones que existen entre fenómenos naturales. Permite describir nuevos hechos o datos, relaciones o leyes, en cualquier campo de conocimiento humano.

\section{Resultados}

Se dice que este método surge empíricamente a la par del origen del hombre, la naturaleza curiosa del mismo le obliga a repetir varios acontecimientos que le dejan cierto conocimiento sobre el funcionamiento y las consecuencias de estos eventos. En Grecia miles de años más tarde, se erige entre los hombres uno llamado Aristóteles que "intento un método para lograr conocimientos seguros, se dedicó a organizar investigaciones y a reunir toda la información posible sobre la Historia Natural. $\mathrm{Su}$ método consistió en la acumulación y clasificación de datos, pero la ausencia de 
hipótesis y de experimentación correcta, hace de la ciencia aristotélica un cúmulo de observaciones inconexas." (Junta de Andalucía, 2012).

Galilei unos siglos más tarde, introduce un método experimental basado en la repetición y la refutación, esta última usada para corregir varios errores científicos y dogmáticos que hasta entonces eran una verdad absoluta. Bacon por su parte aporta al método con la introducción de la variable inductivo-experimental y Lavoisier en cambio agrega herramientas que ayudaran a cuantificar de manera más exacta los resultados obtenidos de los experimentos, así en adelante se añaden más elementos que aportan mayor seriedad y confiabilidad al método.

\section{Fundamentos}

Es importante señalar que, para llevar a cabo la ejecución de un método científico, hay que introducir tres características esenciales como Orden: los fenómenos en la naturaleza ocurren dentro de un orden; Determinismo: aceptamos que cada observación está determinada por un acontecimiento anterior y así sucesivamente; y Comprobabilidad: Cada interrogante en un proceso puede ser explicado y comprobado (Castán, 2012). De estos devengan procesos para distintas áreas de conocimiento: método Universal usado usualmente en la filosofía como el materialismo dialéctico; métodos generales usados para las ciencias generales con herramientas como Hipotético deductivo, hipotético inductivo, observación, experimentación, medición y otros; y métodos particulares usados para ciencias particulares como Ensayo clínico, intervención Comunitaria (Horsford \& Bayarre, 2009).

En nuestro caso, se debe ampliar la composición de los métodos generales (dentro del cual se centra nuestro tema) en primer orden los métodos empíricos que basan sus estudios en la observación, medición y experimentación básicamente; y los métodos teóricos que centran su atención en el análisis y síntesis, deducción e inducción, hipótesis deductivo-histórica y lógica principalmente.

Hay que tener en cuenta que el desarrollo del método científico debe ser imparcial y desprejuiciado. Las conclusiones se sostienen independientemente del estado mental, creencia religiosa o el estado de conciencia del investigador y/o el tema de la investigación (Deiana, Granados, \& Sardella, 2018). 


\section{Técnicas del Método Científico}

Como ya lo mencionamos anteriormente, para este método es indispensable desarrollar diversas técnicas que posibiliten la comprensión global del tema en cuestión, para ello se aplica las técnicas Hipotético-Deductivo: único método con el que se puede obtener información científica, aplicada a las ciencias formales el procesos comprende Observación, hipótesis, experimentación, teorías; Analítico: proceso cognoscitivo, que descompone un objeto en partes para estudiarlas en forma aislada; Sintético: integra los componentes de un objeto de estudio, para estudiarlos en su totalidad; Histórico-comparativo; Cuantitativo: usa la recolección de datos para probar la hipótesis, con base en la medición numérica y análisis estadístico, para establecer patrones de comportamiento y probar teorías; y Cualitativo: utiliza la recolección de datos, sin medición numérica, para descubrir o afinar preguntas de investigación en el proceso de interpretación (Castán, 2012).

Hay que tomar en cuenta seguir patrones de ejecución que aseguren un desarrollo correcto de la actividad investigativa, Asens \& Parra (2002) consideran que para el desarrollo de un trabajo de investigación son necesarias la realización de tareas de documentación, experimentación y comunicación. En la documentación se procede a recopilar información sobre el conocimiento precedente al tema, la que se debe analizar y utilizar como elemento soporte para el texto. En la etapa de experimentación, se procederá a la recolección, análisis e interpretación de los datos. Las técnicas propias de esta etapa son específicas para los diferentes campos del saber pero que requieren también una disciplina para su ejecución. Finalmente, la comunicación constituye la culminación del trabajo de investigación esta debe ser el resultado de una actuación correcta en cuanto al diseño del texto respetando la estructura del trabajo científico y utilizando las normas de sintaxis y de estilo adecuadas, así como la aplicación de las normas propias de la comunicación oral.

\section{Fases del método científico}

Para continuar, hay que regirse bajo 4 fases o etapas obligatorias: formulación de la hipótesis, explicación de los procedimientos que se van a seguir, acumulación de datos e información y análisis de la información obtenida. Por otra parte Morles (2002) 
considera solo 3 fases generales: Observación previa de la realidad, haciendo énfasis en ciertas características que interesan al investigador y entre las cuales se supone que existen ciertas relaciones de causa-efecto; Construcción de un modelo teórico, es decir, un sistema de hipótesis, o explicaciones altamente probables, sobre la relación entre el conjunto de variables que han sido derivadas de las hipótesis propuestas; y, verificación de las hipótesis con base en la observación controlada del mayor número posible de variables del hecho o fenómeno en estudio. En ambos casos se considera la repetición de la hipótesis como fuente de fiabilidad hasta llegar a una conclusión.

Desde otro enfoque, Asuad \& Vázquez (2014) consideran 4 puntos sustanciales en la ejecución del método científico que parte en la observación científica, esta Implica el uso y análisis de técnicas y uso de instrumentos para registrar comportamientos e identificar atributos del fenómeno estudiado. Luego el experimento científico Implica a que el investigador intervenga activa y deliberadamente en el objeto de investigación para producir los datos que le interesan. Provoca reacciones en dicho objeto para medirlas y analizarlas. La documentación Implica comparar un enunciado o propuesta hipotética con una fuente de información pertinente y de confiabilidad y mediante esta comparación se realiza una inferencia sobre la veracidad o falsedad del enunciado y finalmente muestreo y entrevista que implica recabar datos e información representativos recoger una muestra de una mayoría o población o universo de estudio para contrastar alguna hipótesis que se investiga.

\section{Discusión}

En el desarrollo del anterior punto se puede identificar claramente que el método científico ha ido progresivamente mejorando a la par de que surgen nuevos conocimientos, sin embargo, también se presentan postulados que contrarrestan la fiabilidad de este método, Méndez (2012) propone que uno de los errores más comunes es sobreestimar el uso de la matemática, ya que produce un exceso de confianza, incluso en ocasiones con un papel validador de teorías y conocimientos. (...), otro punto desfavorable es el rechazo a las ciencias sociales, porque no usan matemática; el evitar consideraciones o argumentos teóricos, si no están ligados a las definiciones en expresiones matemáticas, por último, el ignorar el papel del diseño de 
investigación. Es decir, no importa cómo se eligen los elementos de estudio o cómo se miden las variables, mientras el modelo matemático describa la realidad.

Otra de las variables que toman fuerza sobre el método científico es la poca fiabilidad de la intuición y el conocimiento empírico, ¿Qué tan acercado a una realidad general está? Mientras que el empirismo radical afirma que sólo las proposiciones que se obtienen de la experiencia son científicas, y por tanto aceptables como verdaderas, Hume (2016) argumenta por qué es imposible justificar el razonamiento inductivo: ciertamente no puede ser justificado deductivamente, y tampoco puede serlo inductivamente (a partir del éxito de la inducción en el pasado), puesto que se trataría de una justificación circular, es decir se caería en un ciclo infinito de repeticiones y redundancia.

Karl Popper, citado por (Génova \& Gonzáles, 2002) considera también (...) que la inducción no es posible: la inducción no es parte del método científico, la inferencia basada en muchas observaciones es un mito; no podemos aspirar a comprobar la verdad de ninguna teoría científica; las hipótesis científicas no pasan de ser meras conjeturas que son aceptadas provisionalmente hasta que aparece una nueva experiencia que las refuta, y a esto Bungue (2017) añade una cualidad irrefutable, pues considera no limitarse a hallar generalizaciones que se adecuen a los datos, sino intentar explicarlas a base de leyes más fuertes; además considera que puede perfeccionarse mediante la estimación de los resultados a los que lleva y mediante el análisis directo, finalmente infiere en que requiere algún conocimiento previo que pueda luego reajustarse y elaborarse.

En cuanto refiere al problema descriptivo en el método científico Ruiz (2007) afirma que este no se queda con los aspectos externos de los procesos o problemas, sino que trata de descubrir los elementos esenciales que expliquen estas hipótesis empíricas, es decir, incluir algún elemento que pueda generalizar dicho postulado, lo que sólo puede realizarse planteando hipótesis teóricas que, son más generales y en las cuales se destacan aquellas relaciones fundamentales entre los fenómenos. De esto devenga otro problema, Alemán (2012) infiere sobre el carácter abstracto de la información sobre el mundo que la ciencia es capaz de proporcionarnos, todo en razón del flagrante contraste entre el contenido de nuestras percepciones y lo que nos dice la ciencia sobre 
ellas. El conflicto surge al comparar acontecimientos de diferentes órdenes. La ciencia nos proporciona información acerca de las propiedades estructurales (meramente regidas a la ciencia), estas son obviamente muy distintas de las cualidades sensibles que notamos en nuestras percepciones, y es entonces cuando emerge la paradoja.

Cabe explicar que no todos los desaciertos del método científico son provenientes de los fundamentos teóricos, sino también el investigador suele cometer errores que en ciertas ocasiones son imperceptibles, Garzón (2019) empieza por los prejuicios y sesgos que el investigador puede desarrollar inconscientemente, radicalizando en la idea de evitarlos a toda costa, otro error común es tomar algo como de sentido común o decidir que algo es tan lógico que no necesita ser probado. Los científicos deben recordar que todo tiene que ser probado antes de que pueda considerarse una hipótesis sólida, por último, se debe de estimar todos los posibles errores que pueden surgir durante las pruebas. Este aspecto es de los más difíciles de controlar, puesto que como afirma Garzón, somos humanos y relativamente podemos caer en nuestras preferencias, pensamientos y sesgos de manera consciente o inconsciente, fórmula para solucionar ello es abrirse hacia otras corrientes de pensamiento, ampliar el rango de visión de la realidad y sobre todo "ponerse en los zapatos" de hacia quienes se limita nuestro estudio.

Llegar hasta el punto de la moral e introducir una variable "ética" dentro del método científico aún se considera un reto, sin embargo, el padre Stanley Jaki citado por (Zenit, 2011) afirmaba que el cientifismo, que motiva a introducir al método científico dentro de todo ámbito cotidiano denunciaba que como enfoque de la ciencia no reconoce los límites de su propio método. La doctrina según la cual los métodos científicos deben extenderse a todos los dominios de la vida intelectual y moral sin excepción no consideraba que varios acontecimientos cotidianos que podrían ser trascendidos a hacia la ciencia no contaban con la cualidad de medibles cuantitativamente, además se toma en dicha teoría que los únicos conocimientos válidos son los que se adquieren mediante las ciencias positivas. 


\section{Conclusiones}

La investigación tuvo como finalidad conocer que el método científico desde sus orígenes hasta el día de hoy ha tenido una gran evolución, la cual ha pasado por diferentes actores, los mismos que cada vez han ido mejorándola para el desarrollo de una investigación. Podemos decir que el método científico es un paso que nos da a conocer a través de sus técnicas por qué ocurren las cosas, mediante la medición y experimentación entre otras, dando como resultado solución a problemas y satisfaciendo las necesidades humanas, mediante esta investigación realizada y ver como el método científico ha ido mejorando al mundo podemos decir que este proceso es de gran importancia ya que por medio de este se podrá seguir desarrollando los avances en la sociedad, es decir como el avance tecnológico, el desarrollo en medicina y diferentes campos aplicativos y asimismo, así mismo se pudo observar los errores o problemas que pueden ser realizados por parte del investigador, ya que muchas de las veces no se realiza las pruebas necesarias para saber si la investigación está terminada.

\section{Referencias}

Alemán, R. (2012). Alcance y limitaciones del método científico. AUTORES CIENTÍFICO-TÉCNICOS Y ACADÉMICOS, 55-64.

Asens, V., \& Parra, A. (2002). El método científico y la nueva filosofía de la ciencia. Anales de Documentación, 9-19.

Asuad, N., \& Vázquez, C. (2014). Marco lógico de la investigación científica. Obtenido de Universidad Nacional de México: http://www.economia.unam.mx/cedrus/descargas/Metodo\%2oCientifico.pdf

Bungue, M. (1967). La investigación científica: su método y su filosofía. México D.F.: Silgo XXI editores.

Bungue, M. (2017). El Planteamiento Científico. Revista Cubana de Salud Pública, 39.

Canaan, R. (2020). Importancia del Método Científico: 5 Razones Principales. Obtenido de https://www.lifeder.com/importancia-metodo-cientifico/ 
Castán, Y. (2012). INTRODUCCIÓN AL MÉTODO CIENTÍFICO Y SUS ETAPAS. Obtenido de Metodología en Salud Pública: http://www.haykhuyay.com/A1/Generic/ECO1/U1U2/metodoCientifico.pdf Deiana, A., Granados, D., \& Sardella, M. (2018). EL MÉTODO CIENTÍFICO. Obtenido de Introducción a la ingeniería: http://www.fi.unsj.edu.ar/asignaturas/introing/MetodoCientifico.pdf Garzón, A. (2019). El Método Científico: Hipótesis, Modelos, Teorías y Leyes. Obtenido de BSC Designer: https://bscdesigner.com/es/metodo-cientifico.htm Génova, G., \& Gonzáles, M. d. (2002). Cuatro problemas de método científicoexperimental que reclaman la apertura a la inteligencia meta-metódica. Universidad de Navarra, 661-680.

Gonzáles, F. (s.f.). El Método Científico. Revista de la Universidad de Costa Rica, 165169.

González, A. M., \& Hernández Alegría, A. (2014). Positivismo, dialéctica materialista y fenomenología: tres enfoques filosóficos del método científico y la investigación educativa. Actualidades investigativas en educación.

Horsford, R., \& Bayarre, H. (2009). Ciencia e investigación científica. Obtenido de Metodología de la investigación científica: http://files.sld.cu/isss/files/2009/o2/curso-metodologia.pdf

Hume, D. (2016). Investigación sobre el entendimiento humano. México D.F.: GreenBooks.

Junta de Andalucía. (2012). Historia del método científico. Obtenido de Agrega: http://agrega.juntadeandalucia.es/taller/ibregue/05042012/ODE-02a254f8-bc8o3915-a9157a95a53fd889/Historia\%2odel\%20M\%C3\%A9todo\%2oCient\%C3\%ADfico.pdf Labajo, E. (2016). El Método Científico. Obtenido de Universidad Complutense de Madrid: https://www.ucm.es/data/cont/docs/107-2016-02-17El\%20M\%C3\%A9todo\%2oCient\%C3\%ADfico.pdf 
Méndez, I. (2012). Método científico, aspectos epistemológicos y metodológicos para el uso de la estadística. SaberEs, 23-31.

Morles, V. (2002). Sobre la metodología como ciencia y el método científico: un espacio polémico. Revista de Pedagogía, 22-27.

Pollit, D., \& Hungler, B. (1991). Investigación Científica en ciencias de la Salud. México D.F.: Interamericana.

Ruiz, R. (2007). El Método Científico y sus etapas. México D.F.: Interamericana.

Zenit. (2011). Reconocer los límites del método científico, herencia del padre Jaki. Obtenido de Zenit: El mundo visto desde Roma: https://es.zenit.org/articles/reconocer-los-limites-del-metodo-cientifico-herenciadel-padre-jaki/ 